\title{
Creation and visualization of archives of operational RBMKs parameters and VVER reactors
}

\author{
A.M. Zagrebayev ${ }^{1}$, I.Yu. Leveev², V.V. Pilyugin3, S. Ten4 \\ National Research Nuclear University MEPhI, \\ Kashirskoe hwy 31, 115409, Moscow, Russia \\ 1 ORCID: oooo-0003-0576-3587, amzagrebayev@mephi.ru \\ 2 ORCID: 0000-0003-2055-5030, ivan.leveev@gmail.com \\ 3 ORCID: 00oo-0001-8648-1690, vvpilyugin@mephi.ru \\ 4 ORCID: 00oo-0oo1-9279-1732, tenstanislav@email.com
}

\begin{abstract}
This article describes the mathematical apparatus for express analysis of the operational parameters' archive.

The developed algorithms, based on the methods of reducing the dimension of the space of variables and the "Chernoff Faces" method, allow visualizing the dynamics of changes in generalized limiting parameters, as well as visually determining the approximation or intersection of the valid parameters values, which in turn can provide scientific and practical use in improving quality of operational personnel work and analysis of situations requiring additional attention and more detailed analysis.
\end{abstract}

Keywords: Operational Parameters Archive, Scientific Visualization, Data Analysis.

\section{Introduction}

Nuclear power engineering is one of the most important and promising objects of modern methods' application of scientific visualization. There are approaches to using scientific visualization methods both for training $[1,2]$ and for research of complex neutron-physical and thermophysical processes in RBMK-1000 nuclear reactors based on computational models $[3,4]$.

At the same time, the safe operation of powerful RBMK and VVER nuclear power reactors is ensured by the availability of information and computer systems that allow measuring, calculating and controlling the most important parameters of a nuclear power unit [5-8].

This information is partially visualized on the power unit control panel and allows operators to manage a nuclear power unit efficiently and safely.

Moreover, in addition to the functions of direct information support for operative control of technological process, the information and computing system archives the current measured and calculated parameters. This allows to conduct a posteriori analysis of the quality of process control in order to obtain new scientific and practical results.

There is a station-wide data exchange network between the process control systems at the stations. Information from various systems with regular interval is sent to the data storage. Next, the power unit operators analyze the data obtained and adjust the reactor operation.

The files in the data storage are not convenient for analysis, therefore, the task of forming such a data storage arises where the archive would be uploaded containing the values of the main parameters of the power unit operation for the selected sections. The archived information stored in this repository can be used for various research purposes in the future. 
Scientific visualization is currently used in various fields, with an emphasis on realistic images of volumes, surfaces, etc. The goal of scientific visualization is to graphically illustrate scientific data so that scientists can better understand data they can visually evaluate.

Let's list as an example some tasks that require knowledge and analysis of the behavior history of RBMK and VVER power units:

- diagnosis of emergency situations at power unit;

- evaluation of operational staff work quality;

- evaluating of the effectiveness of ongoing measures to improve algorithm and control systems;

- creation of an information database of real data for development of mathematical software for the training apparatus;

- creation of adapting mathematical models of processes, ongoing in nuclear power installation;

- creation of self-organizing programs of “operator advisor" type, using work experience of operational staff in regular and emergency situations (solving problems of artificial intelligence);

- identification of reasons of reactor core's separate items and main technological equipment break down (solving problems of predictive analytics).

\section{Problem definition}

Depending on the problem, requirements for pattern, type, amount of stored information and also for the level of its specification may significantly differ. For example, to solve the problem evaluating the quality of operational personnel, it is necessary to store information on the type and number of monitored parameters, the values of which are beyond the limits established by the regulation, the number and type of operator's actions on the control object (movement of control and protection systems (for CPS), coolant flow rate adjustment, etc.), the degree of spatial stability of the three-dimensional energy release field, etc. When solving the problem of identifying the failure's causes, for example, of fuel elements and a channel, information for the period from several days to several years may be needed. In this case it is of interest a behavior backstory of such parameters as each channel capacity, its power generation, coolant flow rate through the channel, fuel elements' burst can detection system data and integrity control of technological channels [5], linear load on a fuel element, stock before the heat transfer crisis, number of transpositions of fuel cartridges and etc.

The limiting parameters for a VVER-type reactor partially coincide with the above, but also contain significant additions and differences associated with the difference in the design of the reactors. For example, the limiting parameter is the concentration of boric acid, the absence of boiling of the coolant, the pressure in the reactor, etc.

However, despite the fact that the above problems are actually of different types, RBMK and VVER power unit operational parameters archive should enable each problem to be solved and moreover, should be an information database for solving newly arising problems. At the current level of computer systems' development at nuclear power plants, it is possible to organize the storage of all experimental and calculated information with high detail in time over a long period of operation, however, this raises problems with express analysis of a large amount of data. The way out of this situation can be the use of the method of scientific visualization, when the initial analyzed data are assigned to one or another form of its graphic interpretation, which can subsequently be analyzed visually, and the analysis's results of this graphic interpretation are then interpreted in relation to the original data. Scientific visualization is a modern, effective approach to data analysis that allows visualization arrays of data of different nature, abstract or real. Visual information is better perceived and allows to deliver results to the user quickly and effectively. Physiologically, the perception of visual infor- 
mation is fundamental for humans. The success of visualization directly depends on the accuracy of its application, namely, on the precise structuring of the approach and the data itself. A modern storage facility for parameters of a nuclear power unit with an RBMK reactor and a special created module for visualizing archive data in a user-friendly form for viewing the archive database from remote workstations are described in work [9]. This module also serves for easy export of data for subsequent analysis and calculations.

The module interface is presented in Fig. 1. In the main window of the program the current state of the database is analyzed and a list of available "time slices" is generated. The header of the main window displays information about the time of the last update. There is a button on the toolbar to update the list of available "time slices" manually. The cartogram of the parameter selected from the list is displayed in the center of the program window. The program periodically (by default every 30 seconds) automatically updates the list of available "time slices".

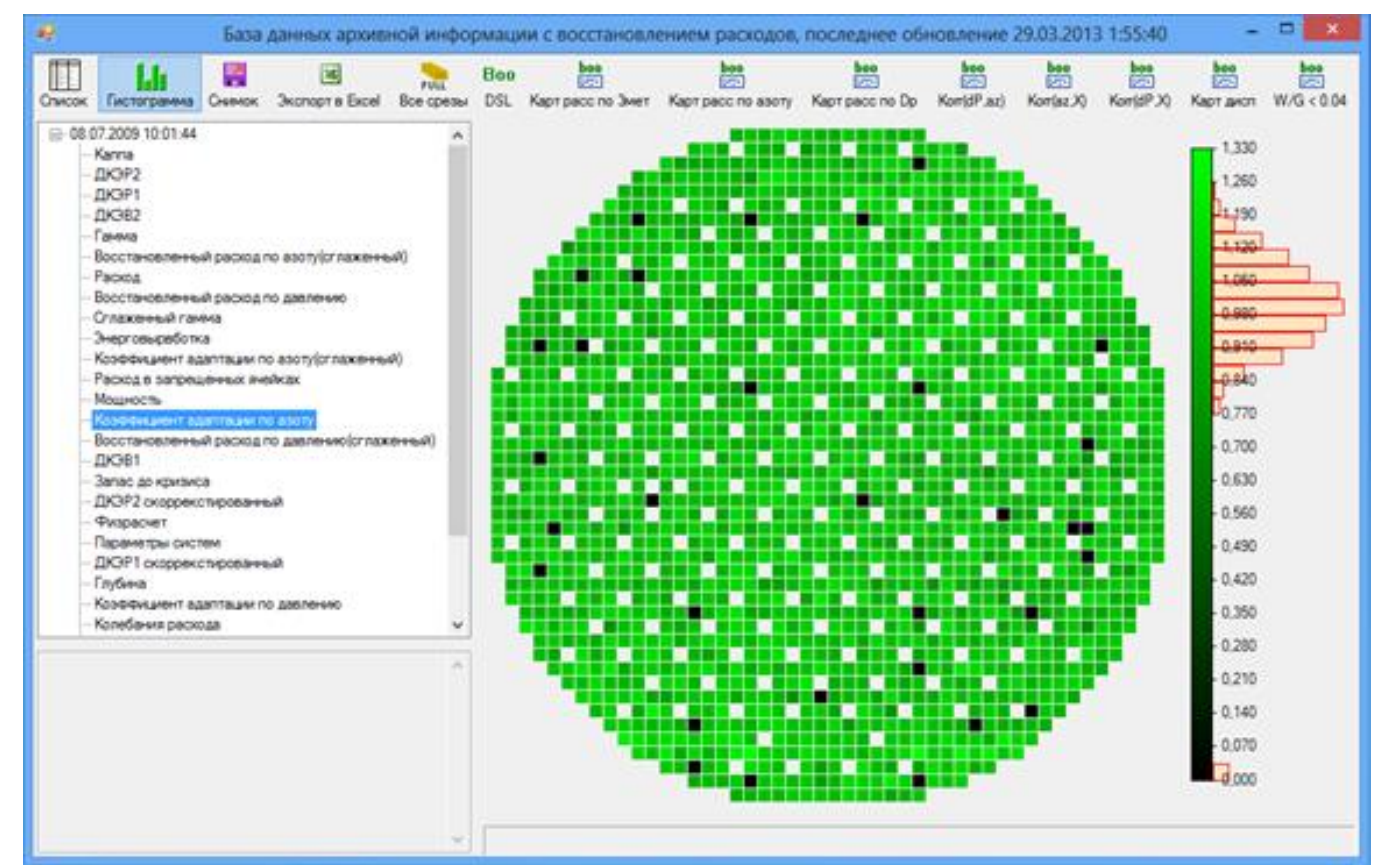

Fig. 1. The window of the visualization module after selecting the required information

The parameters are extracted from the "SKALA-MICRO" system such as core fueling, the coolant flow rate in each channel, information about the position of control rods, the readings of the neutron flux sensors and the estimated parameters reactor power, energy-producing of fuel assembly and reactivity margin. In many ways, we used a similar approach when developing software for creating an archive of operational parameters of a power unit with a VVER-type reactor.

As a result, a software package was created that allows loading archives of VVER reactor parameters into the database and view the already loaded data in a convenient user interface. The display of data on the $3 \mathrm{D}$ core model was optimized by simplifying the cell models.

Two separate visual modules have been developed to display the parameter values: a table and a $3 \mathrm{D}$ model of the VVER reactor core. 


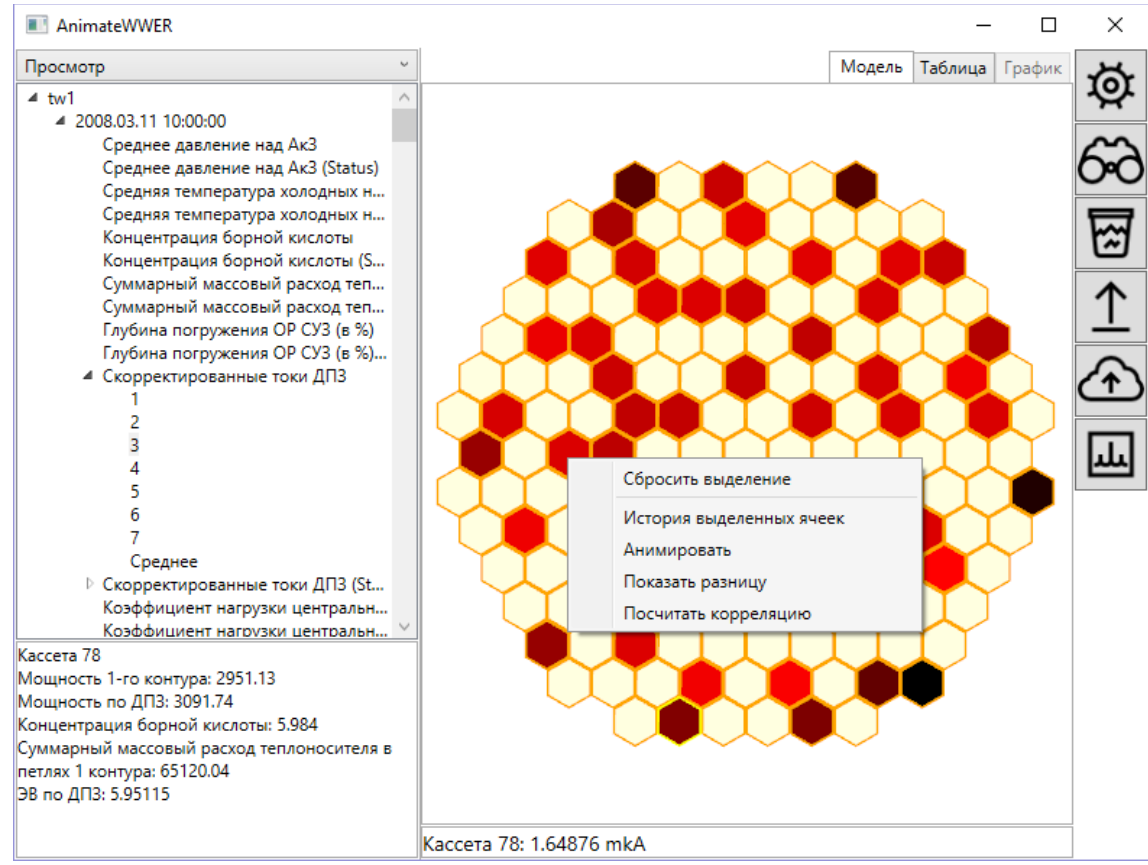

Fig. 2. 2D visualization window after selecting the required information

There is a tree-like display of the database state (a complete set of loaded data) on the left in all modules. The data in the tree view is structured as follows: campaign-slice-parameterlevel (if there are several levels). There is a window below the tree view for displaying extended information on the cell that the mouse is pointing to.

A module for animation of a3D data flow was developed, which allows viewing changes in parameter values throughout the active zone in the form of animation with customizable parameters, a difference module to display the difference in parameter values for two different time slices, a cell value history display module and a parameter value correlation module. A histogram window was also added to display the number of values that fall within a certain interval between the minimum and maximum values for the current display.

The user interface, developed as part of this work, allows loading configuration files into the database, archived data, displaying already loaded configuration and provides a convenient interface for displaying loaded archived data.

Displaying the current configuration, the tree-like display of the archives loaded into the database is updated automatically after any critical actions (loading a new configuration, cleaning the database, loading the archive).

The developed software package supports the expansion of the visualized parameters when receiving a new archive. That is, when receiving an archive that contains other parameters or the same ones but supplemented, it is possible to load this archive into the system without changing the software package itself.
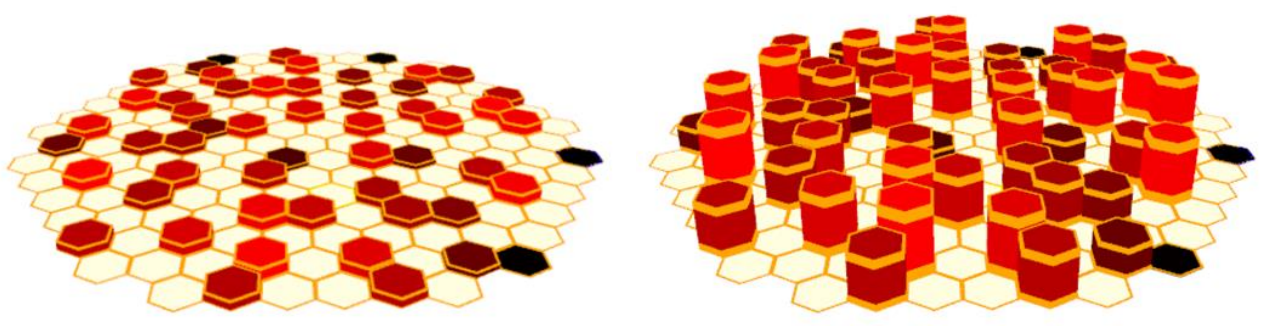

Fig. 3. 3D model of the VVER core

Despite the advantages of the visualization module above, which consist in the presentation of information in the form of cartograms and graphs of detailed behavior of local parameters, 
there are some problems in terms of rapid analysis of the parameters' temporal behavior. It would be desirable to visualize the generalized parameters of a nuclear power unit containing the most important information about the technological process in the Integral.

This opportunity is fundamentally possible, since there are really single objects, for example, neutron fields, graphite masonry temperatures, etc., although they are measured by sensors discretely located in space. This is on the one hand. On the other hand, the archive of operational parameters contains parameters correlated with each other, since different control systems provide dissimilar information but about the same object.

Thus, there is, firstly, a need to compress information and obtain a number of parameters, the physical meaning of which simplifies the visual analysis of the process history, and, secondly, the development (or application) of modern methods of visualization of multidimensional objects and processes.

This work illustrates the proposed approach with specific examples.

\section{Visualization of multidimensional data}

There are frequent cases in which the structural data units are sets of many independent features. In such situations, work is carried out with multidimensional scientific data, which are most often set in the form of tables, where each line corresponds to a separate object, and the elements of the line are separate numerical characteristics of this object.

Since the result of visualization is usually a two-dimensional image, and human consciousness is not capable of perceiving spaces with a dimension higher than three, the image of such data, taking into account all the features, becomes a nontrivial task. Multidimensional data visualizations are usually built from models for $2 \mathrm{D}$ data.

It is proposed to use the least squares method [10] and the method of selecting significant ordinates [11] as the algorithms for compressing information on the neutron field. The first one is used to compress intra-reactor information in space, and the second one is to compress single and integral parameters in time.

Let's briefly consider the technique of compressing information in space. Let us assume that the field of some operational parameter of the reactor core is described by a spatio-temporal function $\Phi(\vec{r}, t)$. Approximate the readings of discretely located sensors is based on the least squares method.

We represent the function $\Phi(\vec{r}, t)$ in the form (1), and seek a solution based on the minimum condition for functional (2).

$$
\begin{gathered}
\Phi(\vec{r}, t)=\sum_{i=1}^{m} B_{i}(t) \varphi_{i}(\vec{r}) ; \\
F=\sum_{i=1}^{m}\left(\Phi\left(\vec{r}_{i}, t\right)-C_{i}\right)^{2} \rightarrow \text { min }
\end{gathered}
$$

here $C_{i}$ is the value of the operational parameter at the points where the sensors are installed; $\vec{r}_{i}$ - coordinates of the reactor core points where the sensors are installed; $\varphi_{i}(\vec{r}), i=1, \ldots, m-\mathrm{a}$ number of linearly independent functions that are selected in advance; $B_{i}(t)$ - time coefficients to be found.

With this formulation of the problem, the solution is obtained in the form

where $\vec{C}=\left(\begin{array}{c}C_{1} \\ C_{2} \\ \ldots \\ C_{N}\end{array}\right)$ is a parameter measurement vector; 
$\vec{B}=\left(\begin{array}{c}B_{1} \\ B_{2} \\ \cdots \\ B_{m}\end{array}\right)$ is a vector of unknown coefficients;

$S=\left(\begin{array}{cccc}\varphi_{1}\left(\vec{r}_{1}\right) & \varphi_{2}\left(\vec{r}_{1}\right) & \ldots & \varphi_{m}\left(\vec{r}_{1}\right) \\ \varphi_{1}\left(\vec{r}_{2}\right) & \varphi_{2}\left(\vec{r}_{2}\right) & \ldots & \varphi_{m}\left(\vec{r}_{2}\right) \\ \ldots & \ldots & \ldots & \ldots \\ \varphi_{1}\left(\vec{r}_{N}\right) & \varphi_{2}\left(\vec{r}_{N}\right) & \ldots & \varphi_{m}\left(\vec{r}_{N}\right)\end{array}\right)$ is a matrix of values of decomposition functions at measurement points.

Thus, instead of the initial $N$ values of the measurement vector $\vec{C}$, m values of the $B_{i}$ coefficients are obtained. The number of $B_{i}$ coefficients is 1-2 orders of magnitude smaller than the number $N$, and satisfactory accuracy is achieved.

The choice of decomposition functions during data compression by the least squares method may be arbitrary in the general case, but in order to achieve maximum approximation accuracy, it is desirable to choose them most closely describing the behavior of the reactor parameters.

On the one hand, such functions are eigenfunctions for solving equations describing the dynamics of the parameters under consideration. On the other hand, finding the exact solution that describes the behavior of the parameters in the reactor is a difficult mathematical task, since a nuclear power reactor is a complex object with distributed parameters.

In [12] there is the following method for experimental determination of the best set of test functions, based on the transition from the initial expansion of the parameters in some functions, that approximately describe their behavior to the canonical expansion obtained from the original.

It is necessary to find such decomposition functions $\left\{\varphi_{i}^{\prime}(\vec{r})\right\}$ in order to present the function $\Phi(\vec{r}, t)$ in the form:

$$
\Phi(\vec{r}, t)=\sum_{i=1}^{M} V_{i}(t) \varphi_{i}^{\prime}(\vec{r}),
$$

in this case $V_{i}$ are not correlated with each other, in contrast to $B_{i}$ in formula (1). $M\left[V_{i} V_{j}\right]=0$ with $i \neq j ; M\left[V_{i} V_{j}\right]=D\left[V_{j}\right]=D_{j}$ with $i=j$, and also $M\left[V_{i}\right]=0$.

The new set of functions is calculated using the following formulas:

$$
\begin{gathered}
\left\{\begin{array}{l}
\widetilde{\Phi}_{i}(\vec{r})=\varphi_{i}(\vec{r})+\sum_{s=i+1}^{N} a_{s i} \varphi_{s}(\vec{r}), i=1, \ldots, N-1 ; \\
\widetilde{\Phi}_{N}(\vec{r})=\varphi_{N}(\vec{r})
\end{array}\right. \\
\left\{\begin{array}{l}
a_{i 1}=\frac{K_{i 1}}{K_{11}} \\
a_{i p}=\frac{1}{D_{p}}\left[K_{i p}-\sum_{\lambda=1}^{p-1} a_{i \lambda} a_{p \lambda} D_{\lambda}\right] \\
i=\overline{2, N} \\
p=\overline{2, i-1}
\end{array}\right.
\end{gathered}
$$

where $D_{p}=K_{p p}-\sum_{\lambda=1}^{p-1}\left(a_{p \lambda}\right)^{2} D_{\lambda}$.

$K_{i j}$ values are correlation moments: $K_{i j}=M\left[\left(B_{i}-m_{i}\right)\left(B_{j}-m_{j}\right)\right]$.

Here $m_{j}=\frac{\sum_{i=1}^{N} B_{j}^{(i)}}{N}, N$ is the sample size.

The desired function $\Phi(\vec{r}, t)$ is expressed as following: 


$$
\Phi(\vec{r}, t)=\sum_{i=1}^{M} m_{i} \varphi_{i}(\vec{r})+\sum_{i=1}^{M} V_{i} \widetilde{\Phi}_{i}(\vec{r})
$$

It is necessary to apply the least squares method described above to find the coefficients.

Due to the fact that the canonical decomposition obtained by the described method allows the best description of the macro-field of reactor parameters, it is advisable to recalculate the functions $\Phi(\vec{r}, t)$ in relation to the dynamic archive in order to achieve the most accurate decomposition, since in this case fewer decomposition functions will be required to fulfil the conditions for the accuracy of decomposition.

This approach to visualizing the archive of operational parameters is illustrated by the following examples.

\subsection{Motion path in principal components}

In this work, the "trajectory of motion" of parameters means a strictly sequential set of points in two- or three-dimensional space with a given frame of reference, where all points are sequentially connected by one continuous line.

The main task is to compress several correlated limiting parameters of the fuel channel by the method of principal components and, subsequently, to visualize dynamically the state in three-dimensional space.

The limiting parameters that ensure the normal operation of the fuel assembly are:

- power of the fuel assembly;

- coolant flow rate;

- power safety factor;

- safety factor for linear load;

- fuel temperature;

- temperature of the shell of fuel elements, etc.

The parameters are projected into the first 3 principal components, which makes it possible to render conventional $3 \mathrm{D}$ visualization using the Principal Component Method.

Further, the "trajectory of motion" of these parameters is visualized for a given time slice in a new space of variables, given by the main components. In fig. 4 shows the trajectories of movement for two different coordinates of the fuel channels, where one time slice corresponds to each point, and the last time slice in a given interval is shown in yellow.
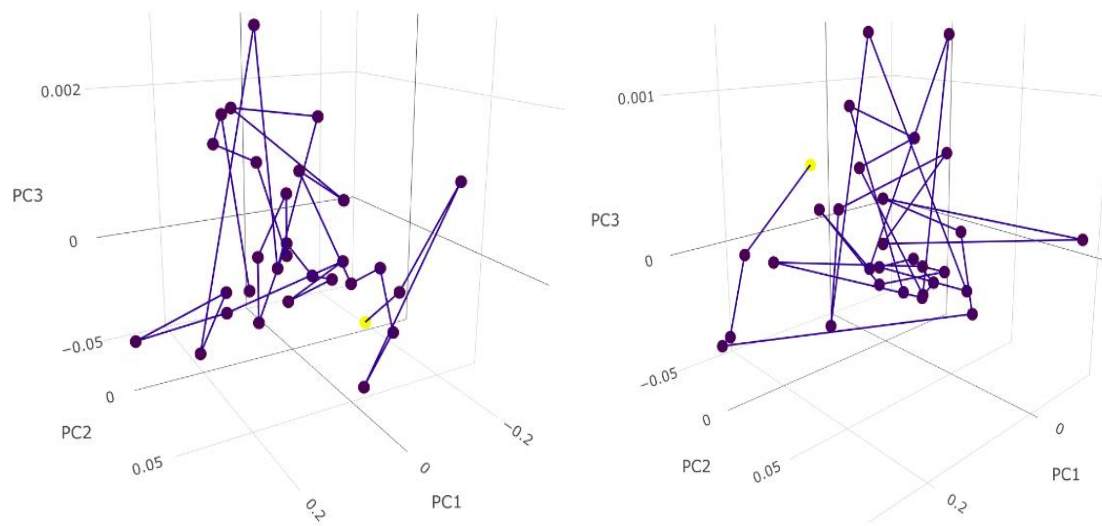

Fig. 4. Motion paths in principal components.

The limits and conditions of safe operation were obtained and projected onto the main components from the technological regulations for the operation of power units, which allows visually noticing whether any of the selected parameters exceeds the maximum permissible value. 
Exceeding the settings of at least one of the parameters leads to an unplanned decrease in emergency power supply. In this work three integral parameters are left as a result of compression that characterize the state of the fuel assembly. This state in dynamics is displayed as the movement in time of a point in three-dimensional space limited by tolerances (Fig. 5).
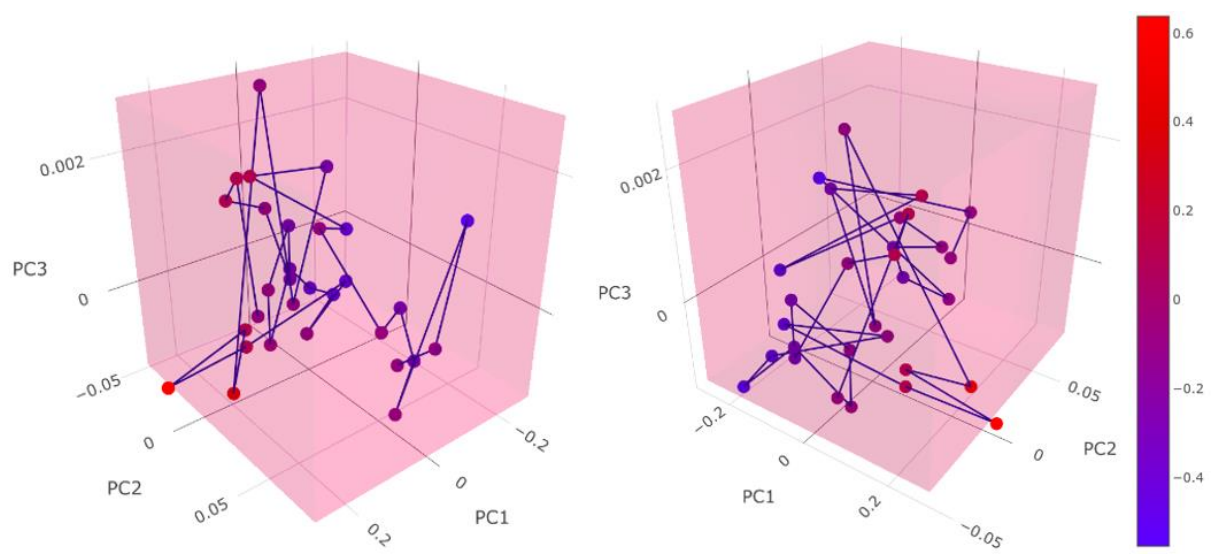

Fig. 5. Visualization of the motion path in the principal components (different angles)

The developed visualization makes it possible to study the behavior of the fuel channel's parameters in time, to reveal deviations, approximation or intersection of the settings for the safe operation of a nuclear reactor, and thereby allows to speed up the process and improve the quality of the archive analysis.

\subsection{Adaptation of the "Chernoff Faces" method to the archive}

"Chernoff Faces" is a convenient approach to parameter behavior visualization invented by Herman Chernoff in 1973 that displays multivariate data in the shape of a human face. The individual parts, such as eyes, ears, mouth and nose represent the values of variables in their shape, size, placement and orientation [13].The idea behind using faces is that humans easily recognize faces and notice small changes without difficulty. Typically, "Chernoff Faces" are used when it is necessary to group (cluster) objects according to several criteria or when it is necessary to analyze presumably complex relationships between variables. Chernoff's method, applied to the express analysis of the operational parameters archive, significantly expands the range of visualized states of the reactor and the power unit as a whole.

As an example, let's use this approach to display power dynamics in local regions of the core. In this case, local regions are understood as quadrants of the reactor core.

Let $W_{(x, y)}^{t}$ be the power of the fuel assembly having coordinates on the core scheme at time $t$ (Fig. 6).

Further, the cartogram of the reactor is divided into 4 parts (quadrants), as shown in Fig. 6. Each quadrant corresponds to a set of channel coordinates located in this quadrant:

$$
\begin{gathered}
Q_{i}=\left\{\left(x_{1}, y_{1}\right),\left(x_{1}, y_{2}\right), \ldots,\left(x_{2}, y_{1}\right),\left(x_{2}, y_{2}\right), \ldots,\left(x_{p}, y_{q}\right), \ldots\right\}, \\
i=1, \ldots, 4-\text { quadrant number }
\end{gathered}
$$




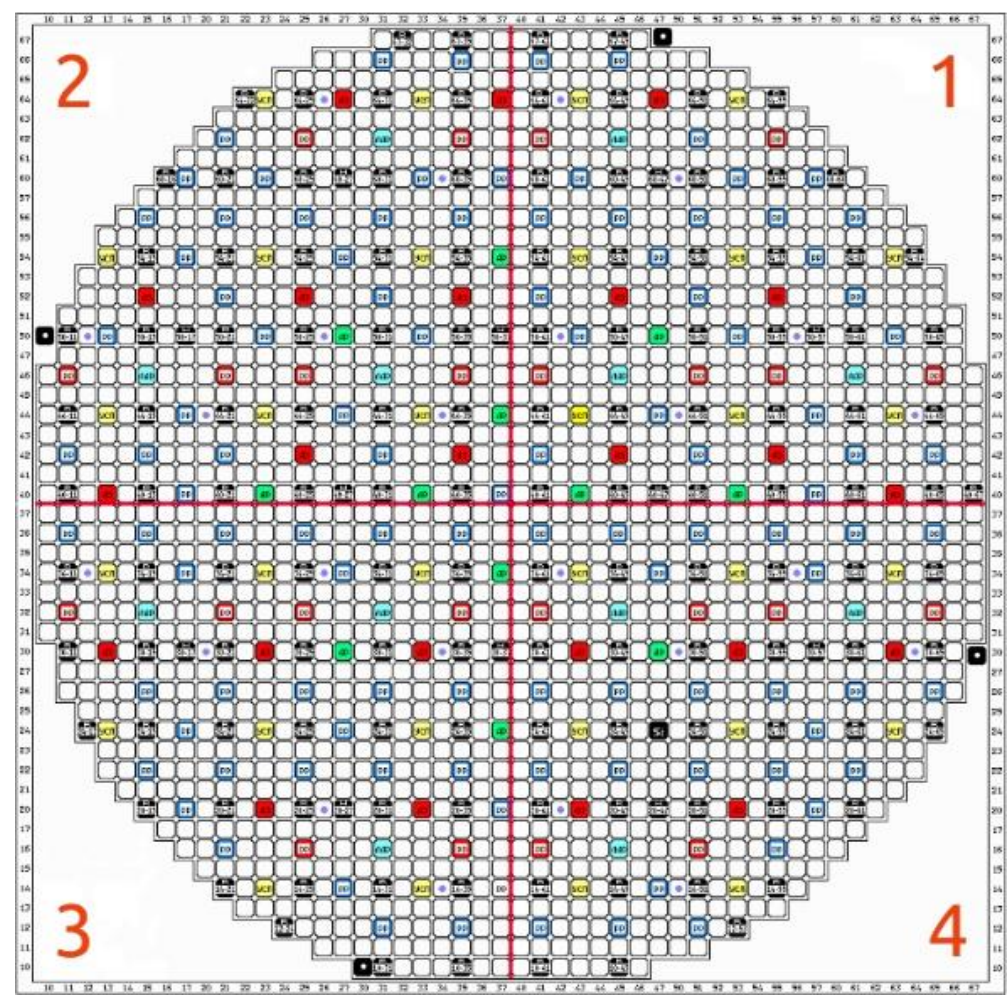

Fig. 6. Scheme of reactor core divided into quadrants

The average power value for each quadrant is taken for each time moment $t$.

$$
\bar{W}_{i}^{t}=\sum_{\left(x_{p}, y_{q}\right) \in Q_{i}} \frac{W_{\left(x_{p}, y_{q}\right)}^{t}}{n},
$$

$n$ - number of channels in quadrant, $i=1, \ldots, 4$ - number of quadrant.

As a result, for each moment of time, each quadrant corresponds to a parameter which is the average value of power $\bar{W}_{i}^{t}$.

Then, the normalization procedure is applied for each quadrant according to the formula for the entire time slice (given time slice):

$$
X_{\text {norm }}=\frac{X-X_{\min }}{X_{\max }-X_{\min }}
$$

$X$ - the initial value is the value at the time $t, X_{\min }$ is the minimum value for the entire time slice, $X_{\max }$ is the maximum value for the entire time slice.

The normalized value is denoted by $\widehat{W}_{i}^{t}$.

At the mapping stage, 4 face characteristics were selected for visualization:

- $\quad P_{1}$ for the tilt of the eyebrows;

- $P_{2}$ for the eye width;

- $P_{3}$ for the length of the nose;

- $P_{4}$ for the bend of a smile.

Each of the characteristics $P_{i}$ lies in the range $[0,1]$. The "average face" is displayed separately, which displays the average values of the parameters for the entire time slice (the specified time slice).Fig. 7 shows an example of such a face the characteristics of which are equal 0.5, i.e.

$$
P_{1}=0.5, P_{2}=0.5, P_{3}=0.5, P_{4}=0.5
$$




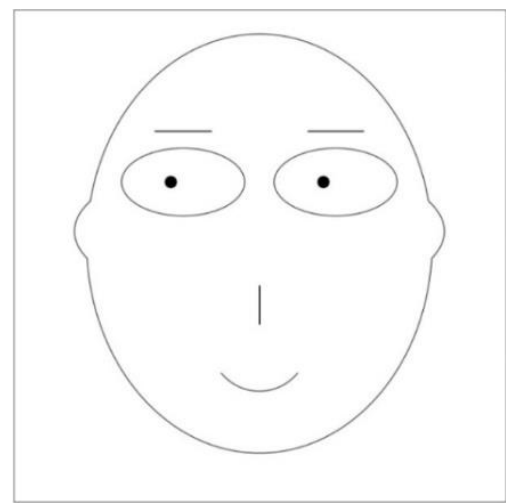

Fig. 7. "Average face"

Further, each characteristic is associated with a normalized quadrant average power value:

$$
P_{i}=\widehat{W}_{i}^{t}
$$

and for each point in time $t$, a corresponding face is displayed.

The final result of power visualization using the "Chernoff Faces" method is shown in Fig. 8.A similar procedure was carried out for the coolant flow rate (Fig. 9).

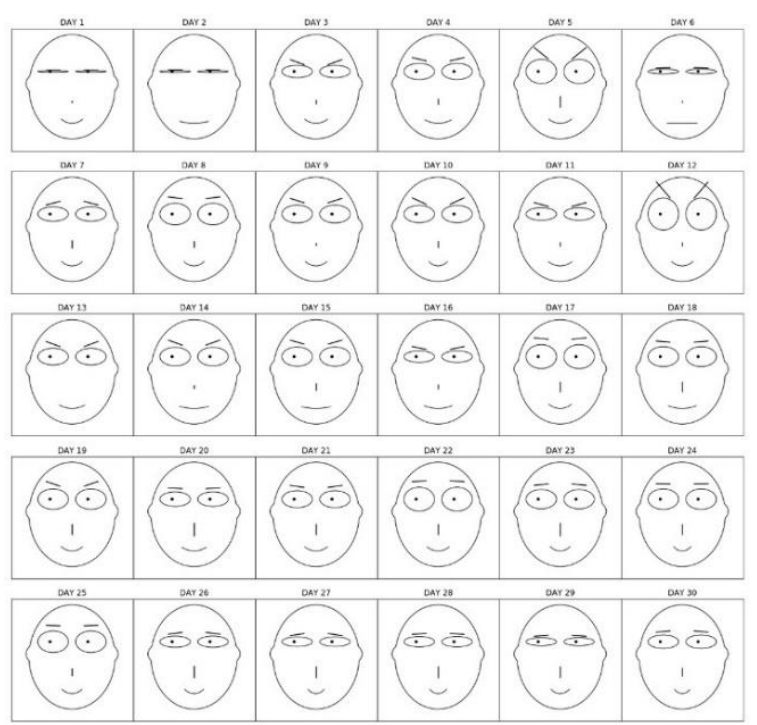

Fig. 8. Visualization results (Power).

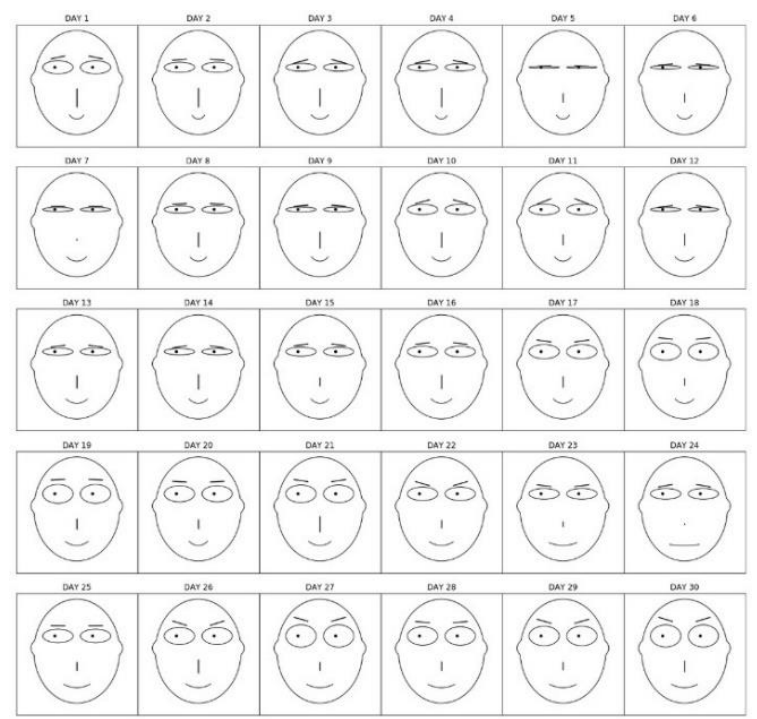

Fig. 9. Visualization results (Flow rate). 
It is possible, analyzing the obtained results, to draw conclusions when some of the parameters deviate strongly from their average values, when maximums or minimums are reached, or implicit relationships between the parameters (when visualizing more parameters) can be found. The result obtained at this stage is intermediate and is used as a demonstration of possible options for visualizing the archive. Such an approach, when using a larger number of parameters, would allow to find visually highlighted clusters of similar facial expressions or detect hidden dependencies between parameters.

\subsection{Temporal Networks}

Temporal Networks is another popular method for visualizing structured, dynamically changing data over time.

A temporal network, also known as a time-varying network, is a network which links are active only at certain points in time. Each link carries information about when it is active, along with other possible characteristics such as weight. Time-varying networks are of particular relevance to spreading processes, like the spread of information and disease, since each link is a contact opportunity and the time ordering of contacts is included [14].

Currently, work is underway to study the visualization methods that are acceptable for use in relation to the existing archive, including the Temporal Networks method that can be used to visualize the interaction of parameters in time and can help to understand, predict and optimize the behavior of system. Representation of data in the form of graphs of vertices connected by edges could reveal new non-trivial patterns of changes in parameters, possible correlations, and also could be used for clustering. At the same time, it becomes possible to assess how local patterns interact and produce global behavior, which is one of the main tasks of archive analysis.

Nowadays, Temporal Networks is actively used in the scientific community, at the same time, there is already a corresponding software for working with dynamically changing data, and there are many articles about application of this method to various data.

\section{Conclusion}

This article describes the mathematical apparatus for express analysis of the archive of operational parameters. The developed algorithms based on the methods of reducing the dimension of the variable space and the "Chernoff faces"method allow visualizing the dynamics of changes in generalized limit parameters, as well as visually determine the approximation or intersection of the parameters of the maximum permissible values. This, in turn, can provide scientific and practical benefits in improving the quality of operational personnel and analyzing situations that require additional attention and more detailed analysis.

Currently, a computer software package has been developed on the basis of the proposed methods that provides flexible customization of the desired visualization. The program module includes components for data export and processing, two-dimensional and threedimensional visualization with the specified settings. The software is implemented with the use of modern effective data analysis tools and provides convenient user interaction functionality.

The proposed software can be used both by the NPP operational staff as an auxiliary tool for improving the efficiency of monitoring the operation of the power unit, and for the purpose of analyzing the existing archival database.

It is planned to continue working to improve the quality and stability of the developed software package, as well as expanding the functionality and adding new features.

\section{References}

1. G. Tikhomirov, I. Saldikov, E. Malikova, L. Kuchenkova, V. Piliugin. Opyt NIIAU MIFI v razrabotke $\mathrm{i}$ ispolzovanii programmnykh sredstv vizualizatsii v uchebnom protsesse v ob- 
lasti iadernykh energeticheskikh ustanovok. [NRNU MEPhI experience in the development and use of visualization software in the educational process in the field of nuclear power plants.] Scientific Visualization, 2012, V.4, Num. 2, pp. 57-63

2. O. Bukalin, A. M. Zagrebayev, V. N. Samanchuk. Vizualizatsiia protsessa neitronnofizicheskogo rascheta iadernogo reaktora. [Visualization of the process of neutronphysical calculation of a nuclear reactor.] Scientific Visualization, 2020, V.12, Num. 1, pp. 112-119

3. Ivanov, N. SHCHukin, S. Bychkov, I. Moiseev, V. Druzhinin, IU. SHmonin. Ispolzovanie sredstv vizualizatsii dlia analiza statisticheskikh oshibok rascheta metodom Monte-Karlo pokanalnykh funktsionalov reaktora RBMK-1000. [The use of visualization tools for the analysis of statistical errors in the Monte Carlo calculation of channel-by-channel functionals of the RBMK-1000 reactor.] Scientific Visualization, 2012, V.4, Num. 1, pp. 22-30

4. D. Zinakov. REQT programma vizualizatsii i analiza rezultatov raschetov polnomasshchtabnykh zon reaktorov RBMK-1000.[REQT is a program for visualizing and analyzing the results of calculations of full-scale zones of RBMK-1000 reactors.] Scientific Visualization, 2012, V.4, Num. 1, pp. 31-41

5. Dollezhal' N.A., Emel'yanov I.Ya. Kanal'nyi yadernyi ehnergeticheskii reaktor. [Channel Nuclear Power Reactor] Atomizdat Publ., 1980.

6. M.A. Abramov, V.I. Avdeev, E.O. Adamov et al.Edited by Yu.M. Cherkashova. Kanal'nyi yadernyi ehnergeticheskii reaktor RBMK.[Channel Nuclear Power Reactor RBMK] GUP NIKIEhT Publ., 2006. 632 p.

7. Ovchinnikov F.Ya., Golubev L.I., Dobrynin V.D., Klochkov V.I., Semenov V.V., Tsybenko V.M. Ehkspluatatsionnye rezhimy vodo-vodyanykh ehnergeticheskikh yadernykh reaktorov. [Operational modes of water-cooled nuclear power reactors] Atomizdat Publ., 1975.

8. Nuclear Power Engineering. Problems. Solution / Edited by M.N. Strikhanov. - Part 1. M.: Social Forecasts and Marketing Center, 2011. - 424 p.

9. A.M. Zagrebayev, R.N. Ramazanov, Nuclear Reactor RBMK Archive Data Visualization. Scientific Visualization, 2015, Q.2, V.7, Num. 2, pp. 1-11

10. Yurova L.N., Naumov V.I., Savander V.I., Zagrebayev A.M. Kompaktnoe predstavlenie vynutrireaktornoi informatsii o potoke neitronov / Fizika yadernykh reaktorov. [Compact representation of in-reactor neutron flux information. / Physics of nuclear reactors.] Atomizdat Publ., 1975, n.4, pp.19-23

11. Alimov A.L., Shchadilov A.E. Optimal'noe adaptivnoe szhatie tsifrovykh soobshchenii po algoritmu kusochno-lineinoi approksimatsii. [Optimal adaptive compression of digital messages using piecewise linear approximation algorithm] Avtometriya Publ., 1983, №3, pp.14-18.

12. A.M. Zagrebayev, V.A. Nasonova, N.V. Ovsyannikova. Matematicheskoe modelirovanie yadernogo reaktora pri sluchainykh vozmushcheniyakh tekhnologicheskikh parametrov. [Mathematical modeling of a nuclear reactor with random perturbations of technological parameters] NRNU MEPhI Publ., 2009. - 400 p.

13. Wikipedia contributors. Chernoff face. Wikipedia, The Free Encyclopedia. Retrieved May 5, 2020. Available at: https://en.wikipedia.org/w/index.php?title=Chernoff_face \&oldid $=958878449$

14. Wikipedia contributors. Temporal network. Wikipedia, The Free Encyclopedia. Retrieved August 11, 2020. Available at: https://en.wikipedia.org/w/index.php?title=Temporal _network\&oldid $=960041057$ 